\section{Radio-opaque punctate opacities on the chest radiograph following intravenous injection of a bismuth compound}

Doreen J Addrizzo-Harris, Andrew Churg, William N Rom

\begin{abstract}
The case history is described of a patient who presented with small rounded punctate metallic opacities widely dispersed on the chest radiograph with accumulation of metallic particles in the right ventricle. Energy dispersive $x$ ray spectroscopy of alveolar macrophages identified a major predominant peak as bismuth. The patient had been injected with a health tonic in Honduras two years earlier.

(Thorax 1997;52:303-304)
\end{abstract}

Keywords: bismuth, metallic opacities.

Bismuth compounds have been used as medical agents since 1733 when bismuth salve was first introduced, followed in 1799 by their use for dyspepsia. ${ }^{1}$ Bismuth subnitrate paste was promoted in 1910 as a treatment modality for chronic abscesses. ${ }^{2}$ The paste was injected directly into cavities and its distribution documented with the developing radiographic technology. Currently, the most commonly used bismuth agents worldwide are bismuth subsalicylate (BSS, Pepto-Bismol, The Proctor and Gamble Company, Cincinnati, Ohio, USA) and colloidal bismuth subcitrate (CBS, De-Nol, Gist Brocades, Delft, The Netherlands). BSS is reported to be in more than $60 \%$ of homes in the USA and more than 10 billion doses have been consumed. ${ }^{1}$ Bismuth compounds have been reported to have adverse effects on various organ systems. ${ }^{3-6}$ Between 1973 and 1980 neurotoxicity was described in approximately 1000 cases in Europe and Australia following large doses $(700 \mathrm{mg}$ to 20 $\mathrm{g} /$ day) of bismuth subnitrate or bismuth subgallate given for long periods (four weeks to 30 years) for gastrointestinal conditions. ${ }^{7}$ Only four case reports of neurotoxicity to BSS have occurred - all in patients who took amounts above the recommended dosage. ${ }^{4-6}$ Given that more than $99 \%$ of the bismuth in an oral dose of BSS is excreted in the faeces, these reports suggest that diffuse colonic abnormalities may result in enhanced absorption through a diffusely abnormal mucosa. We describe an unusual case, not previously reported, of intravenous bismuth exposure in a 39 year old patient who presented with metallic opacities on his chest radiograph.
Case report

A 39 year old man from Honduras who emigrated to the USA two years previously sought medical attention for evaluation of headache, myalgias, weight loss of $4.5 \mathrm{~kg}(10 \mathrm{lb})$, intermittent abdominal pain, back pain, and cough. He had been well until he was injected intravenously in his left forearm while in Honduras two years previously, after which he was ill for several days with a flu-like illness. $\mathrm{He}$ denied any other medical problems including a previous history of venereal disease. He was not taking medications and had no known allergies. He was an ex-smoker with a 20 pack year history who had stopped two years previously. He had taken cocaine in the past but denied any intravenous drug use. He had been employed in the agricultural industry picking fruits and vegetables for more than 10 years and had worked for eight months in a jewellery store, and had no inhalational exposures. Blood evaluation revealed normal serum electrolytes including a normal creatinine of $1.1 \mathrm{mg} / \mathrm{dl}$. The haemogram was within normal limits except for mild thrombocytopenia of 131000 cells $/ 1$. Arterial blood gas tension on room air showed a pH of $7.43, \mathrm{PaCO}_{2}$ of $37 \mathrm{~mm}$ torr and a $\mathrm{PaO}_{2}$ of $100 \mathrm{~mm}$ torr with $98 \%$ saturation. The chest radiograph revealed multiple punctate $1 \mathrm{~mm}$ metallic densities scattered throughout the lung fields (fig 1) and lining the contour of the right atrium and ventricle (fig 2). A computed tomographic scan of the chest revealed discrete $1 \mathrm{~mm}$ punctate dense opacities scattered in both lungs with a subpleural distribution. Pulmonary function testing revealed forced vital capacity (FVC) of 5.141 (116\% of predicted), forced expiratory volume in one second $\left(\mathrm{FEV}_{1}\right)$ $3.861(105 \%)$, total lung capacity (TLC) 7.091 (109\%), and a lung transfer factor (TLCO) adjusted to haemoglobin of $139 \%$ predicted. Fibreoptic bronchoscopic examination was normal. Bronchoalveolar lavage recovered $14.6 \times 10^{6}$ cells with $87 \%$ macrophages, $10 \%$ lymphocytes, 3\% neutrophils, and no eosinophils. Histological examination of the transbronchial biopsy specimen revealed normal bronchial wall and lung parenchyma. Alveolar macrophages were evaluated for particles by morphology and energy dispersive $x$ ray spectroscopy. Most of the particles gave a single peak for bismuth. Rare particles of iron, titanium, and iron-chromium were also present.

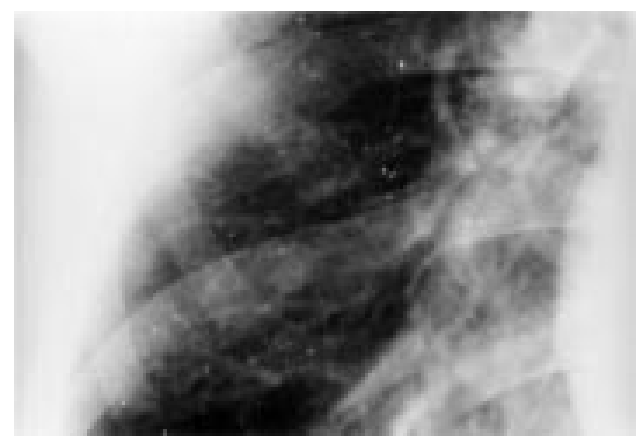

Figure 1 Posterior-anterior chest radiograph showing punctate $1 \mathrm{~mm}$ metallic densities scattered throughout the lung fields. 


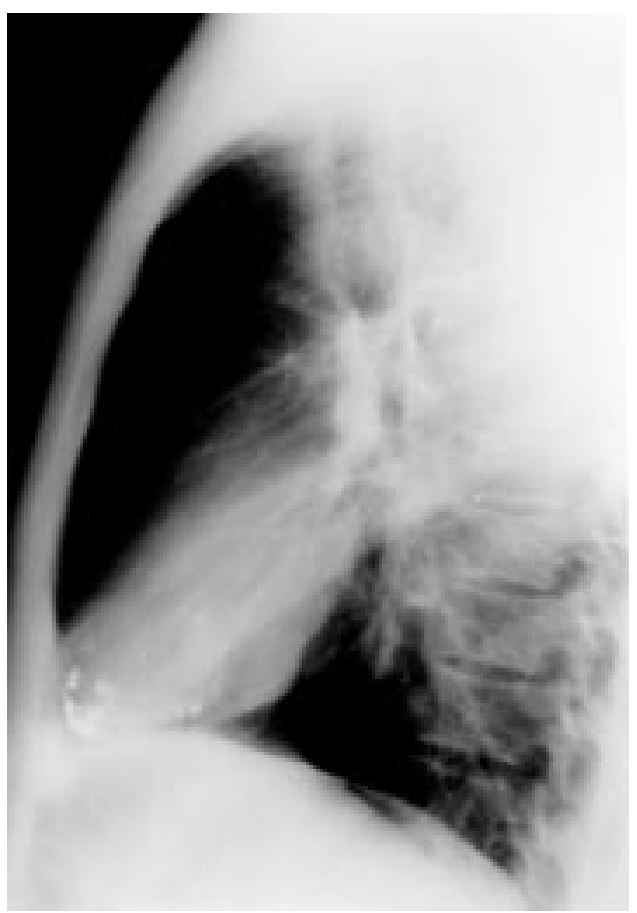

Figure 2 Lateral view showing metallic densities in the right ventricle.

\section{Discussion}

Analysis of particles from alveolar macrophages recovered by bronchoalveolar lavage in this case revealed numerous particles of bismuth. Takemura et al evaluated inorganic particles in lavaged cells from 43 non-smokers, exsmokers, and normal volunteers ${ }^{8}$ without identifying bismuth. Rom and colleagues used transmission electron microscopy and energy dispersive $x$ ray spectroscopy to determine the particle burden in 42 dust exposed subjects and non-exposed controls. ${ }^{9}$ Individuals with dust exposure had a fourfold increase in the numbers of particles per alveolar macrophage compared with controls but, again, bismuth was not found in either group. Johnson et al performed a similar analysis on lavage fluid from 12 patients with occupational exposure and 10 with no known history of dust exposure ${ }^{10}$ without finding bismuth. Churg and Steven ${ }^{11}$ examined both lung parenchyma and airway mucosa from 12 non-smokers and 10 smokers. Although particle concentrations for both types of tissue were in the range of 106 particles/g, with particles of iron and titanium as well as a host of other known ambient atmospheric contaminants commonly observed, no bismuth particles were seen. These studies indicate that bismuth particles are not normally found in the lungs of either the general population or those with most types of common occupational exposures. Thus, the presence of numerous bismuth particles in the alveolar macrophages and the finding of extremely radiodense nodules on chest radiographic examination suggest that the substance with which this patient was injected was bismuth. Finally, the patient's systemic symptoms were unlikely to be related to the intravenous injection of a bismuth compound since bismuth toxicity is associated with encephalopathy. ${ }^{4-7}$

The authors thank Natalie Little for editorial assistance and Dr $\mathrm{H}$ William Harris for referral of this patient. This study was supported by NIH MO1 RR00096.

1 Bierer DW. Bismuth subsalicylate: history, chemistry, and safety. Rev Infect Dis 1990;12(Suppl 1):S3-8.

2 Beck EG. Bismuth paste in chronic suppurations. St Louis: C V Mosby, 1910.

3 Lambert JR. Pharmacology of bismuth-containing compounds. Rev Infect Dis 1991;13(Suppl 8):S691-5.

4 Mendelowitz PC, Hiffman RS, Weber S. Bismuth absorption and myoclonic encephalopathy during bismuth subsalicylate therapy. Ann Intern Med 1990;112:140-1.

5 Gordon MF, Abrams RI, Rubins DB, Barr WB, Correa $\mathrm{DD}$. Bismuth subsalicylate toxicity as a cause of prolonged encephalopathy with myoclonus. Mov Disord 1995:10: encephar

6 Jungreis AC, Schaumburg HH. Encephalopathy from abuse of bismuth subsalicylate (Pepto-Bismol). Neurology 1993; of bismuth 1265 .

7 Australian Drug Evaluation Committee. Adverse effects of bismuth-subgallate. Med f Aust 1974;2:664-6.

8 Takemura T, Rom WN, Ferrans VJ, Crystal RG. Morphological characterization of alveolar macrophages from phological characterization of alveolar macrophages from individuals with occupational exposure to in

9 Rom WN, Churg A, Leapman R, Fiori C, Swyt C. Evaluation of alveolar macrophage particle burden in individuals occupationally exposed to inorganic dusts. 7 Aerosol Med 1990;5:S43-56.

10 Johnson NF, Haslam PL, Dewar A, Newman-Taylor M Identification of inorganic dust particles in bronchoalveolar lavage macrophages by energy dispersive x-ray microanalysis. Arch Environ Health 1986;41:133-44.

11 Churg A, Stevens B. Mineral particles in the human bronchial mucosa. II. Cigarette smokers without emphysema. Exp Lung Res 1992;18:687-714. 